ARTICLE

https://doi.org/10.1038/s41467-019-10303-z

\title{
Electrosynthesis of high-entropy metallic glass nanoparticles for designer, multi-functional electrocatalysis
}

\author{
Matthew W. Glasscott (10) ', Andrew D. Pendergast (i) 1, Sondrica Goines (1) 1, Anthony R. Bishop (D) 1,
} Andy T. Hoang (1) ${ }^{1}$, Christophe Renault (10 ${ }^{2}$ \& Jeffrey E. Dick (1) ${ }^{1,3}$

Creative approaches to the design of catalytic nanomaterials are necessary in achieving environmentally sustainable energy sources. Integrating dissimilar metals into a single nanoparticle (NP) offers a unique avenue for customizing catalytic activity and maximizing surface area. Alloys containing five or more equimolar components with a disordered, amorphous microstructure, referred to as High-Entropy Metallic Glasses (HEMGs), provide tunable catalytic performance based on the individual properties of incorporated metals. Here, we present a generalized strategy to electrosynthesize HEMG-NPs with up to eight equimolar components by confining multiple metal salt precursors to water nanodroplets emulsified in dichloroethane. Upon collision with an electrode, alloy NPs are electrodeposited into a disordered microstructure, where dissimilar metal atoms are proximally arranged. We also demonstrate precise control over metal stoichiometry by tuning the concentration of metal salt dissolved in the nanodroplet. The application of HEMG-NPs to energy conversion is highlighted with electrocatalytic water splitting on CoFeLaNiPt HEMG-NPs.

\footnotetext{
${ }^{1}$ Department of Chemistry, The University of North Carolina at Chapel Hill, Chapel Hill, NC 27599-3290, USA. ${ }^{2}$ Laboratoire de Physique de la Matière Condensée, Ecole Polytechnique, CNRS, IP Paris, 91128 Palaiseau, France. ${ }^{3}$ Lineberger Comprehensive Cancer Center, School of Medicine, The University of North Carolina at Chapel Hill, Chapel Hill, NC 27599-3290, USA. Correspondence and requests for materials should be addressed to J.E.D. (email: jedick@email.unc.edu)
} 
C omplex cocktails of dissimilar metals alloyed into a single phase may present advantageous physicochemical properties, such as enhanced tensile strength ${ }^{1}$ and increased catalytic activity ${ }^{2}$. Only recently have groups started to alloy five or more elements together into a new class of materials, termed high-entropy-alloys (HEAs) $)^{3,4}$. HEAs are generally defined as containing five or more near-equimolar principle components alloyed into a crystalline solid-solution phase stabilized at an elevated mixing entropy. While the enthalpy of mixing favors phase separation to form intermetallic microstructures, materials with a homogeneous distribution of atoms can be left in a high entropy state under certain experimental conditions ${ }^{5-9}$. Unique properties arising from the proximal arrangement of dissimilar metal atoms, often referred to in terms of interatomic d-band ligand effects ${ }^{10}$ or crystal lattice strain ${ }^{11}$, are of great interest for generating advanced structural and functional materials. While these crystalline materials have been the subject of significant investigation ${ }^{12-15}$, disordered amorphous materials containing five or more components, termed High-Entropy Metallic Glasses (HEMGs), may also offer access to properties arising from dissimilar metal interactions, though these complex materials remain largely unexplored ${ }^{16,17}$.

Generally, high-entropy materials are formed by thermal approaches in which compositionally favorable pure metal ingots are liquefied, homogenized, and rapidly cooled to generate the bulk solid material ${ }^{18}$. Translating these planar bulk materials into nanoparticles (NPs) maximizes active surface-area-to-volume-ratio, which directly impacts electrocatalytic efficiency ${ }^{19}$. Though applying high-entropy catalytic nanomaterials to energy-relevant reactions holds great potential, the fabrication of HEA-NPs by a complex thermal-shock method was only recently reported ${ }^{20}$. The application of such high temperatures using these methods necessitates the use of thermally resistant substrates and may incur stoichiometric inconsistencies due to the differences in vapor pressure between molten metals. In contrast to thermal approaches, electrodeposition of metal salt precursors onto a conductive surface constitutes a room-temperature, scalable approach for the formation of amorphous metallic glass films composed of $\mathrm{NPs}^{21,22}$. In addition, the active surface area of electrodeposited NPs may be further enhanced by generating porous or rough particles $^{23}$. For cathodic alloy electrodeposition experiments, variability in NP coverage, size, and stoichiometric composition stems from three main issues: preferential precursor nucleation and growth on energetically favorable sites leading to uneven surface coverage, diffusion layer overlap between neighboring NPs resulting in NP size polydispersity, and variation in precursor electrodeposition potential causing some metals to electrodeposit at higher atomic concentrations.

We recently demonstrated a solution to the issues outlined above by confining metal salt precursors to water nanodroplets suspended in dichloroethane (DCE), allowing for the isolated delivery of a specific number of precursor salt molecules to a localized nucleation and growth domain upon nanodroplet collision with a conductor 24,25 . In this method, termed nanodropletmediated electrodeposition, delivery of the precursor atoms to the substrate results from the formation of a $\sim 10 \mathrm{~nm}$ droplet/electrode contact radius upon nanodroplet collision, which we recently exploited to observe the nucleation and growth of single Pt NPs in real time on ultramicroelectrodes (UMEs) ${ }^{26}$. In these experiments, we determined that elevated mass transfer (i.e., rapid reduction of chloroplatinate to $\mathrm{Pt}^{0}$ ) within sub-femtoliter $\left(10^{-15} \mathrm{~L}\right)$ nanodroplets played an integral role in the formation of porous Pt NPs. Interestingly, a 55-ms carbothermal-shock followed by rapid quenching $\left(10^{5} \mathrm{~K} / \mathrm{s}\right)$ played an integral role in the first reported fabrication of HEA-NPs by $\mathrm{Hu}$ et al. in $2018^{20}$.
Extending this concept, here we use nanodroplet-mediated electrodeposition to demonstrate that the collision of nanodroplets with a biased electrode represents an electro-shock event on the order of $100 \mathrm{~ms}$, rapidly reducing up to eight confined metal salt precursors into HEMG-NPs with precisely tunable stoichiometric ratios. Furthermore, we use this method to design a CoFeLaNiPt HEMG-NP electrocatalyst for complete water electrolysis to demonstrate the multi-functional potential of this synthetic method.

\section{Results}

Electrosynthesis and characterization of HEMG-NPs. Figure 1a shows a representative collision of a single nanodroplet filled with $8 \mathrm{mM} \mathrm{CoCl}_{2}, \mathrm{MnCl}_{2}, \mathrm{CrCl}_{3}, \mathrm{NiCl}_{2}$, and $\mathrm{VCl}_{3}$ (40 mM of metal salt in total) onto a carbon fiber $\operatorname{UME}\left(r_{\mathrm{UME}}=4 \mu \mathrm{m}\right)$. The minimal background current provided by the UME due to its micrometer dimensions facilitates the observation of single nanodroplet collision events. After an initial sharp rise corresponding to the collision of the nanodroplet with the UME, the current decays due to the consumption (electrolysis) of the metal salt initially contained inside the nanodroplet ${ }^{27,28}$. Importantly, the width of the cathodic peak indicates that electrodeposition is finished within ca. $100 \mathrm{~ms}$, a timescale similar to the one encountered in the carbothermal-shock synthesis $(55 \mathrm{~ms})$. The amount of charge transferred during the reduction process, $Q_{\text {red }}$, is obtained by integrating under the blip-type response shown in Fig. 1a. The analysis of multiple independent cathodic peaks leads to an average value of $Q_{\text {red }}=3.47 \pm 2.68 \mathrm{pC}$. This amount can be compared to the initial amount of metal precursor contained within a nanodroplet and the corresponding charge, $Q_{\text {ini, }}$ required to reduce all the precursor ions. According to Faraday's Law, this charge is given by Eq. 1 .

$$
Q_{\text {ini }}=\frac{4}{3} \pi F r_{\text {drop }}^{3} \sum_{i=1}^{k} n_{i} C_{i}
$$

where $F$ is Faraday's constant, $r_{\text {drop }}$ is the average radius of a nanodroplet, $n_{\mathrm{i}}$ is the number of electrons involved in electron transfer for the salt $i$, and $C_{\mathrm{i}}$ is the concentration of the salt $i$. Using an average value of $r_{\text {drop }}=450 \mathrm{~nm}$, obtained by previous dynamic light scattering and nanoparticle tracking analysis results $^{26}$, we calculated a value of $Q_{\text {ini }}=3.53 \mathrm{pC}\left(Q_{\mathrm{red}} / Q_{\text {ini }}=\right.$ $98.2 \%)$. We conclude that all the metal ions initially contained in the nanodroplet are effectively reduced upon collision with an electrode biased at $-0.4 \mathrm{~V}$ vs. $\mathrm{Ag} / \mathrm{AgCl}$ (Fig. 1b). For the remainder of this work, we use a higher cathodic potential $(-1.5$ $\mathrm{V}$ vs. $\mathrm{Ag} / \mathrm{AgCl}$ ) to ensure that electrodeposition occurs at or near the mass transfer limit for each depositing species.

Transmission electron microscopy (TEM) was used to investigate the microstructure of binary CoNi NPs electrodeposited at equal stoichiometric ratios (Fig. 1c), giving a selected area electron diffraction (SAED) pattern with diffuse rings characteristic of amorphous materials ${ }^{29,30}$. Probing the atomic distribution within the amorphous microstructure by energy dispersive X-ray spectroscopy (EDX) revealed a disordered cobalt-nickel arrangement. In this work, we demonstrate an important feature of the electro-shock synthesis: precise tuning of NP stoichiometry can be achieved by modulating the ratio of the confined metal salt within water nanodroplets. By adding $20 \mathrm{mM} \mathrm{CoCl}_{2}$ and $20 \mathrm{mM}$ $\mathrm{NiCl}_{2}$ to the nanodroplets and analyzing the composition of the resulting NPs electrodeposited onto highly oriented pyrolytic graphite (HOPG) at $-1.5 \mathrm{~V}$ vs. $\mathrm{Ag} / \mathrm{AgCl}$ with $\mathrm{EDX}$ and inductively coupled plasma mass spectrometry (ICP-MS), we confirmed the stoichiometry of $\mathrm{Co}_{0.5} \mathrm{Ni}_{0.5}$ was in agreement with the initial stoichiometry of the metal salt dissolved in the nanodroplet. Tuning the proportion of $\mathrm{CoCl}_{2}$ and $\mathrm{NiCl}_{2}$ facilitated the generation of $\mathrm{Co}_{0.25} \mathrm{Ni}_{0.75}$ and $\mathrm{Co}_{0.75} \mathrm{Ni}_{0.25}$ 
a

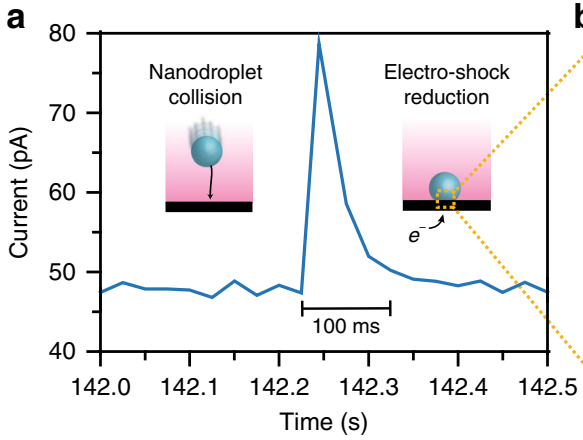

b

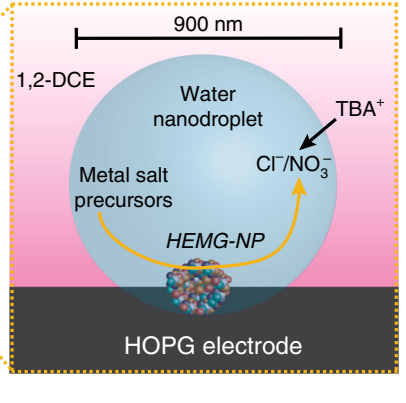

d

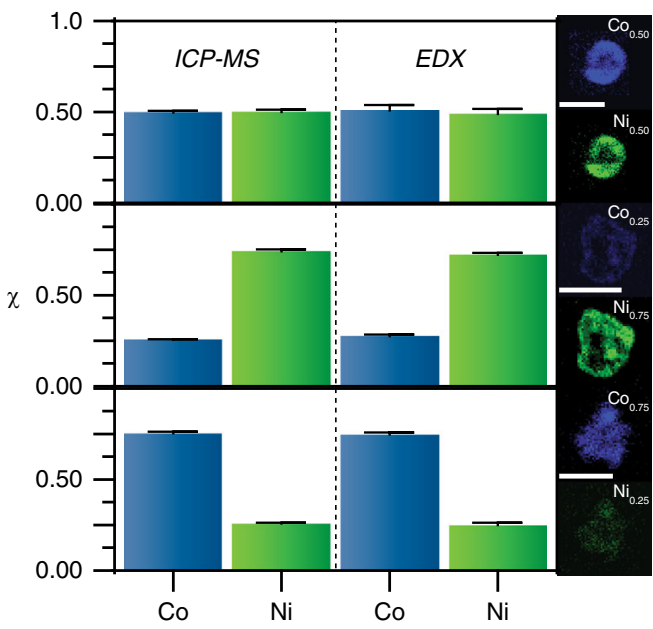

e
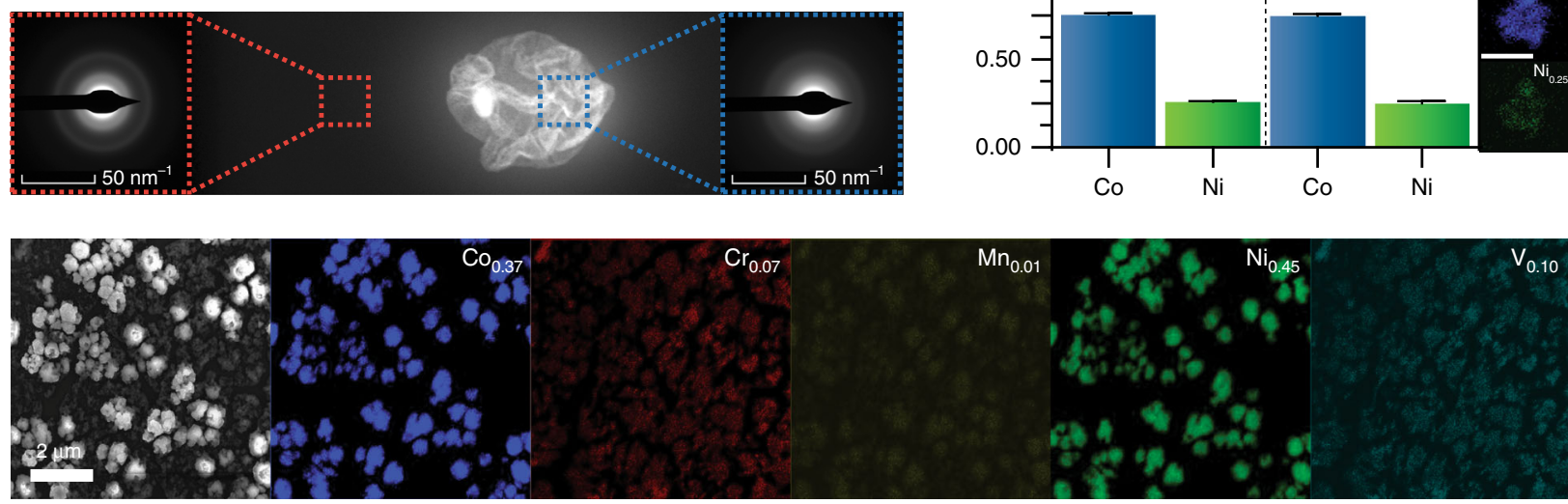

Fig. 1 Nanodroplet-mediated electrodeposition overview for controlling NP stoichiometry and microstructure. a Current transient corresponding to the collision of a single nanodroplet onto a carbon fiber UME $\left(r_{\mathrm{UME}}=4 \mu \mathrm{m}\right)$ biased at $-0.4 \mathrm{~V} \mathrm{vs}$. Ag/ $\mathrm{AgCl}$. Nanodroplet contents are fully $(>98 \%)$ reduced within $100 \mathrm{~ms}$, facilitating disordered co-deposition of various metal precursors. b Representation of a nanodroplet collision event highlighting the rapid NP formation at the water/substrate interface and the charge balance ensured by the transfer of $\mathrm{TBA}^{+}$across the oil/water interface. c An amorphous microstructure is confirmed by a lack of crystallinity at high resolution and the presence of diffuse rings on the SAED pattern. $\mathbf{d}$ Correlated ICP-MS and EDX results on Co0.5Ni0.5, Co0.25Ni0.75, and Co0.75NiO.25 MG-NPs confirming precise control over NP stoichiometry. e Alloy film electrodeposited from aqueous solution of equimolar metal salt precursors demonstrating phase and stoichiometric heterogeneity. Source data for panels $\mathbf{a}$ and $\mathbf{d}$ are provided in the Source Data file

MG-NPs with high precision, which were also verified by ICP-MS (Fig. 1d, Supplementary Table 1). Importantly, ICP-MS data were perfectly aligned with EDX quantification despite the greater uncertainty in EDX measurements ${ }^{31}$, as indicated in Fig. 1d. These results stand in stark contrast to the stoichiometric variation within a $\mathrm{CoCrMnNiV}$ film electrodeposited from an aqueous solution containing $20 \mathrm{mM}$ aliquots of each precursor metal salt onto a glassy carbon electrode biased at $-1.5 \mathrm{~V}$ vs. $\mathrm{Ag} / \mathrm{AgCl}$. The resulting EDX maps reveal a preferential codeposition of a CoNi-rich phase and separate $\mathrm{CrMnV}$ phases (Fig. 1e). Using nanodroplet-mediated electrodeposition, the stoichiometry of the CoCrMnNiV NPs can be controlled to near-equimolar ratios, as shown by EDX and validated by ICPMS, maximizing the mixing entropy (Supplementary Fig. 1). Typical electrodeposition and electroplating methods employ chemical additives, such as polyethylene glycol or hydrochloric acid, to obtain a desirable microstructure, which must be optimized based on the metal salt composition ${ }^{32}$. In this generalized method, confining metal salts to water nanodroplets appears to mitigate the phase-separation effects, as NPs formed by electro-shock reduction maintain the disordered structure and intended stoichiometry shown by EDX and ICP-MS over a wide variety of metal compositions (vide infra). It is worth noting that typical lithographic methods used to generate complex alloy nanomaterials incur up to $50 \%$ variability in stochiometric control $^{33}$, whereas this method allows precise stochiometric control within $2-5 \%$. Furthermore, the ICP-MS results validate EDX as a robust semi-quantitative method for determining approximate NP stoichiometries. These results demonstrate the successful electrosynthesis of disordered MG-NPs and are in agreement with the literature for the formation of mixed-metal oxide films of $\mathrm{CoNi}^{34,35}$. Alloys in this work are discussed in terms of the base metal without regard for the oxidation state (i.e. CoNi). X-ray photoelectron spectroscopy (XPS) data found in Supplementary Figs. 2-3 detail oxidation states of metals in CoFeLaMnNi and CoFeLaNiPt HEMG-NP systems, revealing that all metal species exist as oxides under the conditions necessary for XPS analysis. While these data are useful in indicating the presence and ambient oxidative condition of the HEMG material, they do not necessarily represent the oxidation state of these materials under an applied bias in solution. We are currently investigating the in situ oxidation states of NPs using surface-interrogation scanning electrochemical microscopy 36,37 .

The scope of the nanodroplet electro-shock method was demonstrated by loading nanodroplets with various combinations of metal salt precursors and showing their atomic arrangement and semi-quantitative stochiometric ratio in the resulting NP by EDX. Figure 2a shows the resulting SEM and EDX images obtained over MG-NPs with up to four elemental components, constituting low-entropy systems with $\Delta \mathrm{S}<1.61 \mathrm{R}^{38}$. Figure $2 \mathrm{~b}$ shows SEM and EDX images obtained for various HEMG-NP systems, which have a calculated mixing entropy $\Delta \mathrm{S}>1.61 \mathrm{R}$. High-angle annular dark-field scanning transmission electron microscopy (HAADF-STEM) and SAED were used to validate the conservation of a disordered, amorphous microstructure in this high-entropy system (Fig. 2c). The high resolution of HAADFSTEM EDX mapping $(<20 \mathrm{~nm})$ showing a disordered atomic arrangement supports the lower resolution EDX images obtained 
a Unary (Fe, Pt)

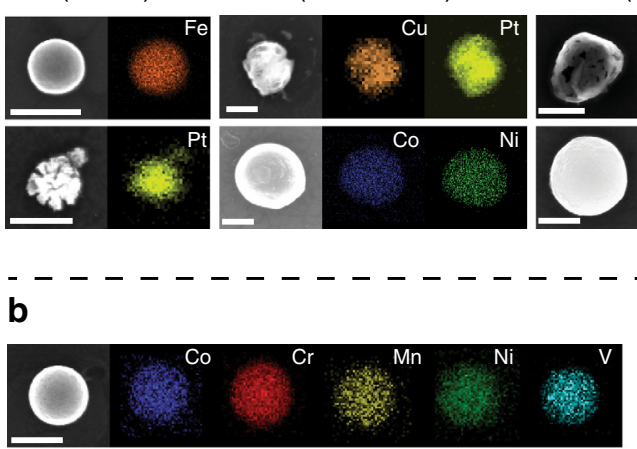

Senary (CoCrLaMnNiV)

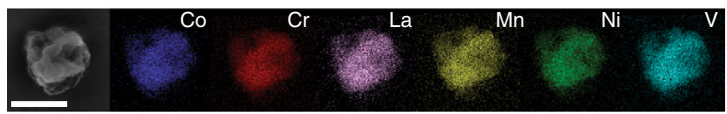

Septenary (CoCrCuGdMnNiV)

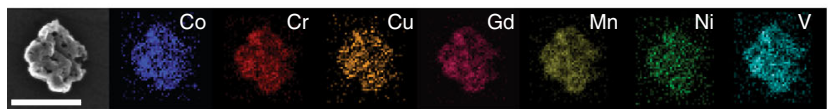

Octonary (CoCrCuGdInMnNiV)

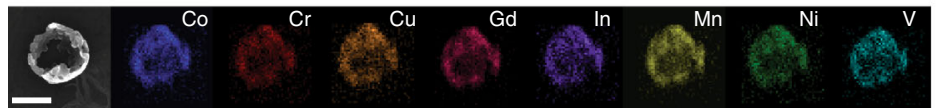

Ternary (CoCrNi, CrCoGd)

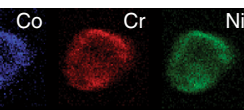

Co
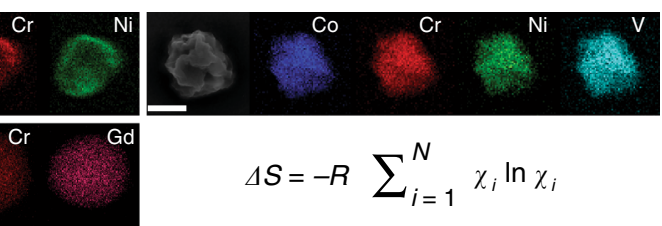

$\Delta S=-R \sum_{i=1}^{N} \chi_{i} \ln \chi_{i}$

Low entropy $(\triangle S<1.61 R)$

High entropy $(\triangle S>1.61 \mathrm{R})$

(CoCrMnNiV

CoCuCrGdNi)

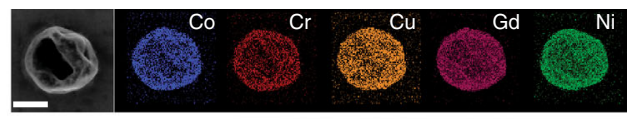

C

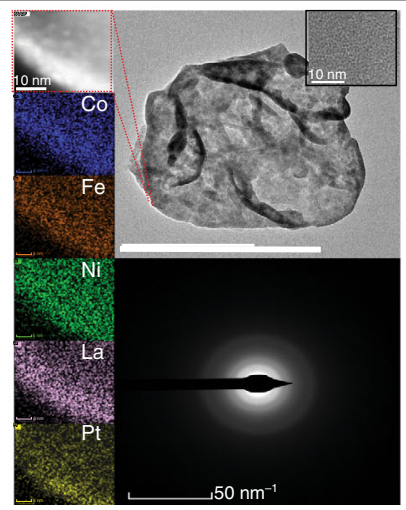

Fig. 2 Elemental and microstructural characterization of alloy NPs with up to eight components. a EDX maps of various low-entropy MG-NPs. A lowentropy classification is dictated by $<4$ elemental components with equimolar stoichiometric ratios to give a $\Delta S<1.61 \mathrm{R}$. $\mathbf{b}$ EDX images of HEMG-NPs $(\Delta \mathrm{S}$ $>1.61 \mathrm{R}$ ) produced by adding up to eight metal salt precursors to the nanodroplets. $\mathbf{c}$ HAADF-STEM images of a CoFeNiLaPt HEMG-NP with accompanying high-resolution EDX images showing disordered elemental distribution at the atomic scale. The diffuse rings on the SAED pattern indicate an amorphous microstructure. All NPs were electrodeposited at $-1.5 \mathrm{~V}$ vs. $\mathrm{Ag} / \mathrm{AgCl}$. Scalebars represent $500 \mathrm{~nm}$ unless otherwise indicated

by SEM. In addition, X-ray diffraction (XRD) was employed to support the amorphous microstructure from SAED (Supplementary Fig. 4). Semi-quantitative atomic percentages via EDX obtained over three particles for each alloy configuration are presented in Supplementary Table 2, confirming maximized entropy and demonstrating stoichiometric control based on the concentration of metal salt precursor added to the nanodroplets.

Some of the metals incorporated into these NPs, such as manganese, have been observed to electrodeposit poorly under general aqueous conditions ${ }^{39}$. Characterization of individual metal salt species electrodeposited on HOPG during cyclic voltammetry $(\mathrm{CV})$ are shown in Supplementary Fig. 5. While these data are useful for determining an approximate onset potential for nucleation and growth onto the carbon substrate, this evaluation may not accurately represent the kinetics of alloy formation due to the previously reported favorable thermodynamic shift associated with co-deposition of multiple metals ${ }^{40}$. Furthermore, our previous results (Fig. 1) comparing nanodroplet-mediated electrodeposition to bulk aqueous electrodeposition, indicate stoichiometric control over systems where precise control has been traditionally difficult to achieve.

Design and application of a multifunctional HEMG-NP electrocatalyst. We demonstrate the versatility of the electro-shock method and its application to designer, multifunctional electrocatalysis by synthesizing a novel CoFeLaNiPt HEMG-NP electrocatalyst for cathodic and anodic water splitting. Generally, electrocatalysts are synthesized and optimized for a particular electrocatalytic reaction. However, it may be economically beneficial to design nanomaterial electrocatalysts amenable to multiple electrocatalytic reactions, a task which is streamlined by the synthetic method presented in this work. For instance, two important reactions for the production of fuel cell reactants are the oxygen evolution reaction (OER) and the hydrogen evolution reaction (HER). Although $\mathrm{Pt}^{41}$ offers facile kinetics for the HER, it displays rather poor performance for the OER. Furthermore, metal oxides $\left(\mathrm{MO}_{\mathrm{x}}\right)$ and oxyhydroxides, such as $\mathrm{CoO}_{\mathrm{x}}{ }^{42}, \mathrm{FeO}_{\mathrm{x}}{ }^{43}$, $\mathrm{NiO}_{\mathrm{x}}{ }^{44}$, and mixed $\mathrm{MO}_{\mathrm{x}}$, such as $\mathrm{NiFeO}_{\mathrm{x}}{ }^{45}$, have been shown to be kinetically favorable toward the OER; however, each of these $\mathrm{MO}_{\mathrm{x}}$ compounds have poor activity toward the HER. Rare-earth oxides, such as $\mathrm{La}_{2} \mathrm{O}_{3}$, have been used to confer stability to materials exposed to alkaline conditions ${ }^{46}$. The literature precedent surrounding these materials inspired us to design a CoFeLaNiPt HEMG-NP electrocatalyst amenable to the OER and the HER at a single nanomaterial. It is important to note that the calculated entropic contribution to the total Gibb's free energy of the NP arises from near-equimolar elemental concentrations, whereas colloquial alloy systems generally contain a single principle element doped with other metals in low concentration ${ }^{47}$. Electrocatalytic activity may be greatly amplified by tuning the stoichiometric ratios, as was reported for the record oxygen evolution activity of $\mathrm{Fe}_{0.25} \mathrm{Ni}_{0.75}$ oxyhydroxide materials ${ }^{48}$. Because the high-entropy classification is predicated on the equimolar ratio of the elemental constituents, it may be necessary to tune metal ratios away from the high-entropy state to optimize electrocatalysis. In this work, it is our goal to explore the nearequimolar synthesis of HEMG-NPs using nanodroplet-mediated electrodeposition and demonstrate the precise tuning of metals rather than to search for an optimal stoichiometric ratio for electrocatalytic water splitting. 


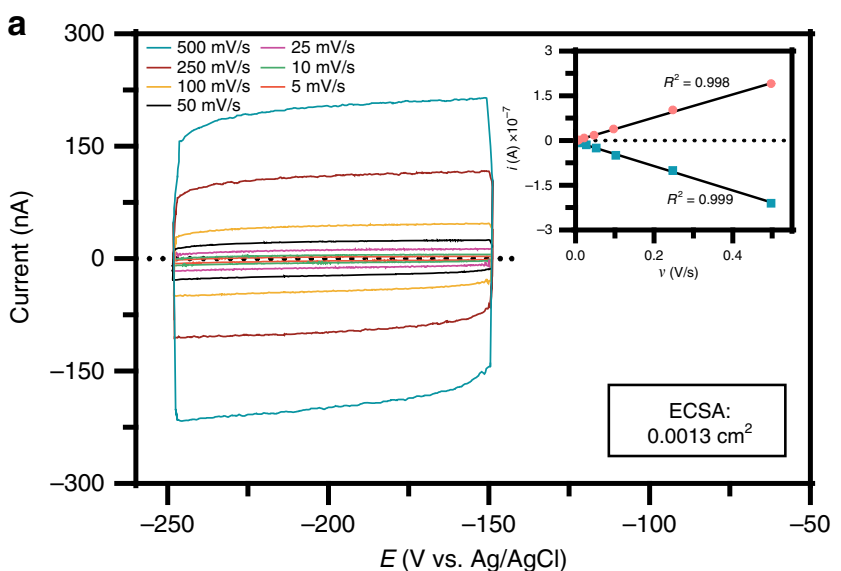

b

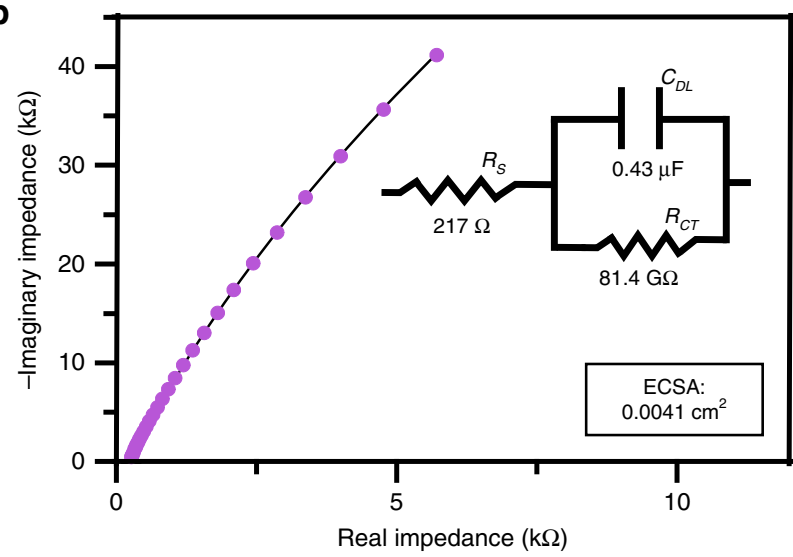

C

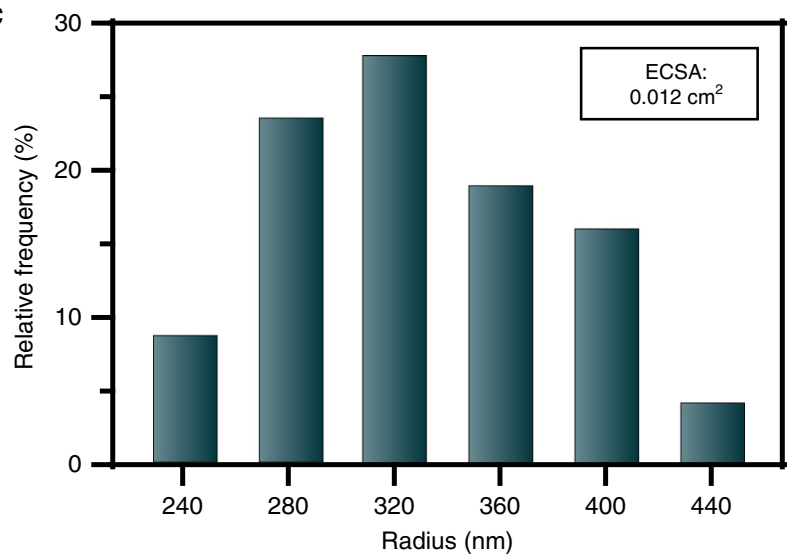

Fig. 3 Evaluation of catalyst electrochemically active surface area. a CV analysis on the CoFeLaNiPt HEMG-NP electrocatalyst from $-0.05 \mathrm{~V}$ to $0.05 \mathrm{~V}$ vs. OCP ( -0.25 to $-0.15 \mathrm{~V}$ vs. $\mathrm{Ag} / \mathrm{AgCl})$ at $5,10,25,50,100,250$, and $500 \mathrm{mV} / \mathrm{s}$ in $0.1 \mathrm{M} \mathrm{KOH}$. The inset plot shows the cathodic and anodic current contributions as a function of scan rate. $\mathbf{b}$ Nyquist plot obtained by EIS fitted to the Randles cell (inset). c Histogram of observed HEMG-NP radius in SEM. The background capacitance of the bare HOPG electrode was subtracted from the obtained double layer capacitance to compensate for the low substrate coverage

Accurate determination of the electrochemically active surface area (ECSA) for a given material is extremely important to quantify and compare electrocatalytic activity. Commonly, the double layer capacitance of the catalyst is determined by $\mathrm{CV}$ in a non-faradaic region or by electrochemical impedance spectroscopy $(\mathrm{EIS})^{49}$. After electrodeposition of CoFeLaNiPt HEMG-
NPs onto the HOPG substrate at $-1.5 \mathrm{~V}$ vs. $\mathrm{Ag} / \mathrm{AgCl}, \mathrm{CV}$ at seven scan rates was conducted (Fig. 3a). Plotting the cathodic and anodic current as a function of the scan rate revealed a linear function, where the slope indicated the double layer capacitance $\left(\mathrm{C}_{\mathrm{DL}}\right)$. EIS can also be used to obtain the double layer capacitance by fitting the impedance response of the system at different frequencies to the Randles circuit (Fig. 3b). The double layer capacitance can be correlated to the ECSA using the specific capacitance of the material, and further details of this analysis can be found in the Materials and Methods. Using CV and EIS, we determined the ECSA of the CoFeLaNiPt HEMG-NPs to be 0.0013 and $0.0041 \mathrm{~cm}^{2}$, respectively. Nanodroplet-mediated electrodeposition offers an additional metric for approximating the ECSA through an integrated form of the Cottrell equation to determine the total number of NPs incident on the electrode surface over a certain deposition time $(t)$, given by:

$$
\text { Coverage }\left(\frac{N P s}{m^{2}}\right)=2 C^{*} D^{\frac{1}{2}} \pi^{-\frac{1}{2}} t^{\frac{1}{2}} N_{A}
$$

where $C^{*}$ is the concentration of nanodroplets suspended in DCE, $D$ is the nanodroplet diffusion coefficient as determined by the Stokes-Einstein relationship, and $N_{A}$ is Avogadro's number. This analysis approximates NP coverage based on diffusional mass transfer of nanodroplets to the electrode surface with subsequent electrodeposition to form a single NP, which was previously validated for Pt NPs electrodeposited from water nanodroplets ${ }^{23}$. By assuming a hemispherical geometry and using the average NP radius of $320 \mathrm{~nm}$ determined by SEM, an approximate ECSA was calculated as $0.012 \mathrm{~cm}^{2}$, as shown in Fig. 3c. For the analysis of our electrocatalytic materials, we used the average of the ECSA values determined by these three methods (Supplementary Figs. 6-17).

We chose to evaluate these electrocatalysts in $0.1 \mathrm{M} \mathrm{KOH}$ due to increased metal oxide stability ${ }^{50}$, reduced cationic interaction $^{51}$, and practicality for real-world water electrolysis applications ${ }^{52}$. Resulting $i R$-corrected linear sweep voltammograms at $10 \mathrm{mV} / \mathrm{s}$ given in Fig. 4 highlight HEMG-NP functionality for both the HER and the OER. Electrocatalyst overpotential $(\eta)$ is most often reported as the difference between the applied potential $(E)$ and the equilibrium potential $\left(E_{\mathrm{eq}}\right)$, which is 1.23 $\mathrm{V}$ vs. RHE for the OER and $0 \mathrm{~V}$ vs. RHE for the $\mathrm{HER}^{53}$. Using this metric at a current density of $10 \mathrm{~mA} \mathrm{~cm}{ }^{-2}$, we obtained an OER overpotential of $377 \pm 4 \mathrm{mV}$ for the HEMG-NPs. Significantly, this finding represents a 139 and $36 \mathrm{mV}$ improvement over unary $\mathrm{Fe}$ and $\mathrm{Ni}$, respectively, demonstrating synergistic transition metal oxide interactions ${ }^{54}$. The HEMG-NP overpotential also corresponds to typically reported values for $\mathrm{IrO}_{x}$, a benchmark OER electrocatalyst ${ }^{55}$. For the HER, the HEMGNPs significantly outperformed the individual components with an overpotential of $555 \pm 2 \mathrm{mV}$, representing an improvement of 132,176 , and $183 \mathrm{mV}$ over $\mathrm{Fe}, \mathrm{Pt}$, and Co NPs, respectively. Though these HER overpotentials are slightly elevated compared to the current literature, the relative activity of each metal component is conserved ${ }^{53}$. Assuming platinum as the active site, this observation may be explained by elemental synergisms between Pt and one or more of the other elemental components on the atomic scale, or possibly an increase in Pt surface area based on its atomic distribution in the amorphous microstructure. Additionally, a bulk polycrystalline Pt disk electrode was evaluated in $0.1 \mathrm{M} \mathrm{KOH}$ to verify the efficacy of the system for the HER, and the obtained overpotential of $201 \pm 15 \mathrm{mV}$ agrees with literature values (Supplementary Fig. 18) ${ }^{53}$. The overpotential necessary to drive the HER on these electrodeposited nanomaterials is significant and possibly indicates active site poisoning. Therefore, while this work demonstrates the potential 


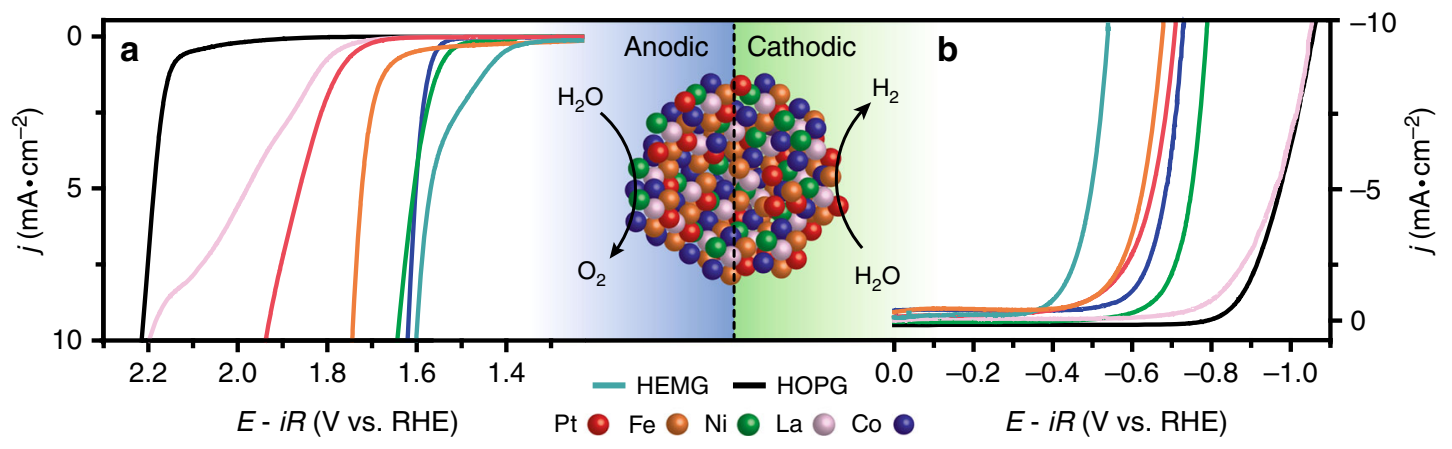

Fig. 4 Electrocatalytic evaluation of a CoFeLaNiPt HEMG-NP electrocatalyst. a Anodic polarization of the HEMG electrocatalyst and its individual components starting from the equilibrium potential of OER, $1.23 \mathrm{~V}$ vs. RHE. Each material was loaded onto the HOPG substrate by nanodroplet-mediated electrodeposition. The current was converted in terms of the current density, j, based on the ECSA. $\mathbf{b}$ Cathodic polarization of the HEMG electrocatalyst and its individual components to drive HER starting from $\mathrm{O} V \mathrm{vs}$. RHE. All measurements were temperature controlled to $25^{\circ} \mathrm{C}$, purged with $\mathrm{O}_{2}$ for $\mathrm{OER}$ and $\mathrm{H}_{2}$ for HER, and iR corrected. For convenience, we represented the OER and HER on the same NP, but these two reactions are not carried out simultaneously. Source data for panels $\mathbf{a}$ and $\mathbf{b}$ are provided in the Source Data file

for the electro-shock method to generate novel electrocatalysts, increasing the overall electrocatalytic efficiency of these materials requires optimization. The HEMG-NP electrocatalyst was found to be stable for at least $1 \mathrm{~h}$ at an overpotential necessary to produce $10 \mathrm{~mA} \mathrm{~cm}^{-2}$ for both the OER and the HER (Supplementary Fig. 19). Furthermore, metal loading on the substrate as a result of the nanodroplet-mediated electrodeposition method was on the order of $10 \mathrm{ng} \mathrm{cm}^{-2}$ for each material, constituting a significant reduction in mass compared to typically employed water splitting electrocatalysts. Other electrocatalytic parameters derived from this analysis, such as mass activity and Tafel slope, can be found in Supplementary Table 3. In this work, it is our intent to showcase a generalized platform for the design and synthesis of novel catalytic multi-component materials. To this end, we present the CoFeLaNiPt material as a successful example of an electrocatalyst specifically designed for both the HER at $\mathrm{Pt}$ active sites and the OER at transition metal active sites, which happens to show interesting synergistic activity above that of its individual components. In principle, the ability to functionally and stoichiometrically tune activity by varying metal salt species and ratios within water nanodroplets offers exciting opportunities for many electrocatalytic systems.

\section{Discussion}

HEMG nanomaterials present an opportunity to enhance a catalyst's chemical and physical properties by selectively incorporating particular elements in certain ratios. In this report, we have presented a facile one-step method, nanodroplet-mediated electrodeposition, to fabricate HEMG-NPs with up to eight principle metallic components while maintaining precise control over elemental stoichiometry. Analysis of single emulsion nanodroplet collision events at a carbon fiber UME indicated complete electrolysis on the order of $100 \mathrm{~ms}$, facilitating rapid NP formation. This electro-shock reduction generated near-equimolar NPs characterized by a disordered, amorphous microstructure confirmed by HAADF-STEM, SAED, and XRD. High-resolution EDX analysis of single NPs demonstrated homogeneous atomic distribution, a property that may elucidate functional synergism towards electrocatalysis. The CoFeLaNiPt HEMG-NP system offers multi-functional activity for the HER and the OER by combining desirable electrocatalytic properties of transition and noble metals, and this generalized synthetic platform represents a unique opportunity to tune this class of HEMG-NP electrocatalysts for a wide range of reactions.

\section{Methods}

Reagents and materials. All chemicals were of analytical grade unless noted otherwise and used as received. Dichloroethane (DCE, 99.8\%), tetrabutylammonium perchlorate (TBAP, 99\%), sodium dodecyl sulfate (SDS, 99\%), hexachloroplatinic acid, and iron (III) chloride were obtained from Sigma-Aldrich and used without further purification. Chromium (III) chloride, cobalt (II) chloride, manganese (II) chloride, vanadium (III) chloride, nickel (II) chloride, indium (III) chloride, cobalt (II) nitrate, nickel (II) nitrate, lanthanum (III) nitrate, iron (II) nitrate, platinum (IV) nitrate, and gadolinium (III) chloride were obtained from Fisher Scientific and used without further purification. Stock solutions (100-300 mM) of each metal were prepared in MilliQ water $(>18 \mathrm{M} \Omega \mathrm{cm})$ and diluted as necessary to be used in the emulsion preparation. Metal salt solutions were stored in a dark refrigerator $\left(4^{\circ} \mathrm{C}\right)$ to avoid photodecomposition. Metal salt compatibility was investigated to prevent competing reactions (Supplementary Table 4). Metal salts were analyzed by UV-vis spectroscopy to ensure no leakage into the DCE phase following sonication (Supplementary Fig. 20).

Instrumentation. The electrodeposition experiments were performed using a CHI model 601E potentiostat ( $\mathrm{CH}$ Instruments, Austin, TX) using the Amperometric $i-t$ Curve technique. Rotating disk electrolysis and electrocatalytic analysis were performed with a WaveDriver 200 and WaveVortex (Pine Instruments,

Durham, NC). A 3-mm radius glassy carbon rod was used as the counter electrode. To decrease the risk of silver leakage from the reference electrode, we employed a double-junction $\mathrm{Ag} / \mathrm{AgCl}$ electrode modified with a home-built $3.5 \mathrm{M} \mathrm{KCl}, 5 \% \mathrm{w} / \mathrm{w}$ agarose salt bridge. No obvious junction potential or other deleterious effects could be observed in the cyclic voltammogram of $1 \mathrm{mM}$ ferrocene methanol in a $0.1-\mathrm{M}$ $\mathrm{KCl}$ aqueous solution as a result of the double-junction reference. In addition, we have found this material to be stable in DCE for over two months. The emulsion was prepared using a Q500 ultrasonic processor (Qsonica, Newtown, CT) with a 1/4" microtip probe. A V-650 UV-vis Spectrometer (Jasco, Oklahoma City, OK) was used to quantify metal salt leakage from the aqueous emulsion phase to the continuous DCE phase. SEM images and EDX spectra were acquired using a Helios 600 Nanolab Dual Beam System (FEI, Hillsboro, OR) and INCA PentaFET -x3 (Oxford, Abingdon, United Kingdom), respectively, at $30 \mathrm{keV}$ and $0.69 \mathrm{nA}$. TEM images and diffraction patterns were collected using a 2010F-FasTEM (JEOL, Peabody, MA) with a zirconated tungsten thermal field emission tip at $200 \mathrm{keV}$. Xray diffraction (XRD) spectra were collected using a Rigaku SmartLab X-ray diffractometer (Rigaku, Tokyo, Japan) in a $2 \theta$ grazing angle configuration. Inductively coupled plasma mass spectrometry (ICP-MS) data were collected using a Nexio 300-D ICP-MS (Perkin Elmer, Waltham, MA). HAADF-STEM and diffraction images were collected with a Talos F200X G2 (FEI, Hillsboro, OR) at $200 \mathrm{keV}$ using a single-tilt holder.

Nanodroplet-mediated electrodeposition procedure. HEMG-NPs were electrodeposited on HOPG or glassy carbon substrate electrodes from a water-in-oil emulsion system, as previously described ${ }^{56}$. In brief, a $30-\mu \mathrm{L}$ aqueous phase containing a total of $40 \mathrm{mM}$ metal salt precursor ions was suspended in a 5-mL DCE continuous phase. The application of a chloroform or ionic liquid continuous phase has also been explored ${ }^{57,58}$. TBAP $(0.1 \mathrm{M})$ was added to the DCE phase as a non-aqueous supporting electrolyte and charge balance mediator. The resultant two-phase solution was subsequently ultrasonicated (500 W, amplitude $40 \%$ ) using a pulse mode method ( $5 \mathrm{~s}$ on, $5 \mathrm{~s}$ off, 6 total cycles) for emulsion formation. Following sonication, a three electrode electrochemical cell was inserted into the emulsion and biased at a potential sufficient to reduce metal salt precursors at the 
electrode surface $(-1.5$ or $-0.4 \mathrm{~V}$ vs. $\mathrm{Ag} / \mathrm{AgCl}, 3 \mathrm{~mm}$ glassy carbon counter electrode). Individual current transients for attoliter nanodroplet collisions were recorded following diffusion and subsequent reduction at a carbon fiber UME. For NP morphology and elemental composition characterization experiments, the electrodeposition was carried out on a 3-mm HOPG disc electrode secured in a Teflon cell with a Viton O-ring. The substrate was exfoliated and rinsed with ethanol prior to emulsion loading, and the post-deposition substrate was rinsed with ethanol and water prior to characterization.

\section{Electron microscopy and energy dispersive spectroscopy analysis. Single} HEMG-NP images and energy dispersive spectroscopy (EDX) spectra were acquired using a Helios 600 Nanolab Dual Beam System (FEI, Hillsboro, OR) and INCA PentaFET -x3 (Oxford, Abingdon, United Kingdom), respectively, at $30 \mathrm{keV}$ and $0.69 \mathrm{nA}$. High resolution elemental mapping for semi-quantitative stoichiometric analysis of individual HEMG-NPs was collected at $30 \mathrm{keV}$ and $11 \mathrm{nA}$ for up to $10 \mathrm{~min}$ of continuous EDX scanning to enhance signal-to-noise ratio while minimizing drift in the SEM. EDX maps were brightness and contrast adjusted and false-colored to demonstrate homogeneous elemental distribution within single HEMG-NPs. Atomic resolution HAADF-STEM/EDX maps were generated using a SuperX Energy Dispersive Spectrometry system (SuperX EDX) with four Silicon Drift Detectors (SDD). Molybdenum grids coated with amorphous carbon (Ted Pella, Redding, CA) were used to support HEMG-NPs for TEM analysis to avoid competing $\mathrm{Cu}$ signal during EDX analysis.

X-ray photoelectron spectroscopy and X-ray diffraction analysis. XPS spectra were obtained under UHV with a base pressure of $5 \times 10^{-9}$ torr on a Kratos Axis Ultra DLD with a monochromatic Al ka source coupled with a hemispherical analyzer. High resolution XPS spectra were baseline subtracted with a Shirley background correction method for subsequent individual peak fitting and peak identification. High coverage HEMG-NP samples were electrodeposited on a glassy carbon rotating disk electrode $(r=2.5 \mathrm{~mm})$ at $1000 \mathrm{rpm}$ and $-1.5 \mathrm{~V}$ vs. $\mathrm{Ag} / \mathrm{AgCl}$ and subsequently rinsed with ethanol and water prior to analysis. High substrate coverage was confirmed via SEM prior to surface characterization by XPS. XPS survey scans indicated the presence of characteristic carbon and oxygen peaks consistent with the underlying glassy carbon substrate electrode. XPS survey scans and high-resolution element scans are presented in the supplementary information for two representative quinary HEMG-NP systems indicating the presence of metal oxide species. XPS peak identification of high resolution regions was achieved by comparing binding energy and peak splitting values with standard literature values. These high coverage samples were subsequently analyzed with a Rigaku Smartlab $\mathrm{XRD}$ in a $2 \theta$ grazing angle orientation to confirm the amorphous microstructure.

Inductively coupled plasma mass spectrometry analysis. Stoichiometric analyses for high coverage HEMG-NP samples were obtained using a Nexio 300-D ICP-MS to supplement EDX analysis for single NPs. High coverage CoNi HEMG-NP samples were prepared by electrodeposition on a glassy carbon rotating disk electrode $(d=5$ $\mathrm{mm}$ ) at $1000 \mathrm{rpm}$ and $-1.5 \mathrm{~V}$ vs. $\mathrm{Ag} / \mathrm{AgCl}$. NPs were extracted with concentrated nitric acid and diluted to $5 \% v / v$ acid matrix in water prior to ICP-MS analysis.

Electrocatalytic analysis. Electrocatalytic analysis was carried out using a Pine WaveDriver 200 and AfterMath software in a temperature-controlled $\left(25^{\circ} \mathrm{C}\right)$ Teflon housing actively purged with $\mathrm{H}_{2}$ (HER) or $\mathrm{O}_{2}$ (OER). The $\mathrm{pH}$ of $0.1 \mathrm{M}$ $\mathrm{KOH}$ was confirmed before each characterization at $13.00 \pm 0.01$. After material deposition, EIS at an AC amplitude of $20 \mathrm{mV}$ was conducted about the OCP from $100 \mathrm{kHz}$ to $10 \mathrm{~Hz}$ in order to determine the double layer capacitance. The uncompensated resistance of the $0.1 \mathrm{M} \mathrm{KOH}$ electrolyte solution was obtained using a pre-programed EIS method and was found to be $217 \pm 29 \Omega$ over 13 experiments (Supplementary Fig. 21). In addition, because the absolute current passed during electrocatalytic evaluation was generally around $50 \mu \mathrm{A}$, the margin of error introduced by this uncompensated resistance amounts to less than $10 \mathrm{mV}$. The uncompensated resistance was accounted for at a correction factor of $85 \%$ for all voltammetric measurements. CVs in the non-faradaic region were then conducted to separately determine the double layer capacitance. Assumptions are made in our treatment of the electrochemically-derived ECSA data including equal conductivity of metals and oxides, negligible pseudo-capacitance, and consistent material specific capacitance, and are therefore suggested in benchmark methods to be accurate to within an order of magnitude of the true ECSA ${ }^{53}$. Due to low substrate coverage, we corrected the ECSA based on the double layer capacitance of the substrate with no electrodeposited material. As an added measure, we also approximated the ECSA using SEM and incorporated each value in the final ECSA determination. Linear sweep voltammograms were then conducted at $10 \mathrm{mV} / \mathrm{s}$ from the equilibrium potential for HER ( $0 \mathrm{~V}$ vs. RHE) or OER $(1.23 \mathrm{~V}$ vs. RHE) to determine catalytic activity. The resulting voltammograms were corrected for $\mathrm{pH}$ and reference electrode by: $E_{\mathrm{RHE}}=E_{\mathrm{Ag} / \mathrm{AgCl}}+0.198+\mathrm{pH}(0.059)$. Each system was evaluated three times to generate statistics on overpotential, Tafel slope, and mass activity, which can be found in the Supplementary Information.

Source data. The source data for Figs. 1a and $4 \mathrm{a}, \mathrm{b}$ are available as source data files.
Received: 4 January 2019 Accepted: 30 April 2019

Published online: 14 June 2019

\section{References}

1. Liu, G. et al. Nanostructured high-strength molybdenum alloys with unprecedented tensile ductility. Nat. Mater. 12, 344 (2013).

2. Stamenkovic, V. R. et al. Trends in electrocatalysis on extended and nanoscale Pt-bimetallic alloy surfaces. Nat. Mater. 6, 241 (2007).

3. Yeh, J.-W. et al. Nanostructured high-entropy alloys with multiple principal elements: novel alloy design concepts and outcomes. Adv. Eng. Mater. 6 299-303 (2004).

4. Cantor, B., Chang, I. T. H., Knight, P. \& Vincent, A. J. B. Microstructural development in equiatomic multicomponent alloys. Mater. Sci. Eng. A 375377, 213-218 (2004).

5. Guo, S. \& Liu, C. T. Phase stability in high entropy alloys: formation of solid solution phase or amorphous phase. Proc. Nat. Sci. Mater. 21, 433-446 (2011)

6. Gludovatz, B. et al. A fracture-resistant high-entropy alloy for cryogenic applications. Science 345, 1153-1158 (2014).

7. Otto, F. et al. The influences of temperature and microstructure on the tensile properties of a CoCrFeMnNi high-entropy alloy. Acta Mater. 61, 5743-5755 (2013).

8. Wang, Y. P., Li, B. S., Ren, M. X., Yang, C. \& Fu, H. Z. Microstructure and compressive properties of AlCrFeCoNi high entropy alloy. Mater. Sci. Eng. A 491, 154-158 (2008).

9. Zhang, W., Liaw, P. K. \& Zhang, Y. Science and technology in high-entropy alloys. Sci. China Mater. 61, 2 (2018).

10. Bligaard, T. \& Nørskov, J. K. Ligand effects in heterogeneous catalysis and electrochemistry. Electrochim. Acta 52, 5512-5516 (2007).

11. Kitchin, J. R., Nørskov, J. K., Barteau, M. A. \& Chen, J. G. Role of strain and ligand effects in the modification of the electronic and chemical properties of bimetallic surfaces. Phys. Rev. Lett. 93, 156801 (2004).

12. Tsai, M.-H. \& Yeh, J.-W. High-entropy alloys: a critical review. Mater. Res. Lett. 2, 107-123 (2014)

13. Gao, M. C., Yeh, J.-W., Liaw, P. K. \& Zhang, Y. High-entropy alloys. (Springer International Publishing, Switzerland, 2016).

14. Pickering, E. J. \& Jones, N. G. High-entropy alloys: a critical assessment of their founding principles and future prospects. Int. Mater. Rev. 61, 183-202 (2016).

15. Senkov, O. N., Miracle, D. B., Chaput, K. J. \& Couzinie, J.-P. Development and exploration of refractory high entropy alloys-a review. J. Mater. Res. 33, 3092-3128 (2018).

16. Greer, A. L. Metallic glasses...on the threshold. Mater. Today 12, 14-22 (2009).

17. Ding, H. Y. \& Yao, K. F. High entropy Ti20Zr20Cu20Ni20Be20 bulk metallic glass. J. Non-Cryst. Solids 364, 9-12 (2013).

18. Miracle, D. B. \& Senkov, O. N. A critical review of high entropy alloys and related concepts. Acta Mater. 122, 448-511 (2017).

19. Reier, T., Oezaslan, M. \& Strasser, P. Electrocatalytic oxygen evolution reaction (OER) on Ru, Ir, and Pt catalysts: a comparative study of nanoparticles and bulk materials. ACS Catal. 2, 1765-1772 (2012).

20. Yao, Y. G. et al. Carbothermal shock synthesis of high-entropy-alloy nanoparticles. Science 359, 1489-1494 (2018).

21. Meng, M. et al. Improved plasticity of bulk metallic glasses by electrodeposition. Mater. Sci. Eng. A 615, 240-246 (2014).

22. Peulon, S. \& Lincot, D. Cathodic electrodeposition from aqueous solution of dense or open-structured zinc oxide films. Adv. Mater. 8, 166-170 (1996).

23. Glasscott, M. W., Pendergast, A. D. \& Dick, J. E. A universal platform for the electrodeposition of ligand-free metal nanoparticles from a water-in-oil emulsion system. ACS Appl. Nano Mater. 1, 5702-5711 (2018).

24. Glasscott, M. W. \& Dick, J. E. Direct electrochemical observation of single platinum cluster electrocatalysis on ultramicroelectrodes. Anal. Chem. 90, 7804-7808 (2018).

25. Glasscott, M. W., Pendergast, A. D., Choudhury, M. H. \& Dick, J. E. Advanced characterization techniques for evaluating porosity, nanopore tortuosity, and electrical connectivity at the single-nanoparticle level. ACS Appl. Nano Mater. 2, 819-830 (2019)

26. Glasscott, M. W. \& Dick, J. E. Fine-tuning porosity and time-resolved observation of the nucleation and growth of single platinum nanoparticles. ACS Nano 13, 4572-4581 (2019).

27. Dick, J. E., Lebegue, E., Strawsine, L. M. \& Bard, A. J. Millisecond coulometry via zeptoliter droplet collisions on an ultramicroelectrode. Electroanalysis $\mathbf{2 8}$, 2320-2326 (2016)

28. Dick, J. E., Renault, C., Kim, B. K. \& Bard, A. J. Simultaneous detection of single attoliter droplet collisions by electrochemical and electrogenerated chemiluminescent responses. Angew. Chem. Int. Ed. 53, 11859-11862 (2014) 
29. McEnaney, J. M. et al. Electrocatalytic hydrogen evolution using amorphous tungsten phosphide nanoparticles. Chem. Commun. 50, 11026-11028 (2014).

30. Delmer, O., Balaya, P., Kienle, L. \& Maier, J. Enhanced potential of amorphous electrode materials: case study of $\mathrm{RuO}_{2}$. Adv. Mater. 20, 501-505 (2008).

31. Friel, J. J. \& Lyman, C. E. Tutorial review: x-ray mapping in electron-beam instruments. Microsc. Microanal. 12, 2-25 (2006).

32. Healy, J. P., Pletcher, D. \& Goodenough, M. The chemistry of the additives in an acid copper electroplating bath: part I. Polyethylene glycol and chloride ion. J. Electroanal. Chem. 338, 155-165 (1992).

33. Chen, P.-C. et al. Polyelemental nanoparticle libraries. Science 352, 1565-1569 (2016).

34. Zaveta, K., Springmann, B. \& Schneider, J. Magnetic aftereffects in amorphous Co- and CoNi-based alloys after field annealing. J. Magn. Magn. Mater. 41, 379-381 (1984).

35. Li, H. et al. Amorphous nickel-cobalt complexes hybridized with 1T-phase molybdenum disulfide via hydrazine-induced phase transformation for water splitting. Nat. Commun. 8, 15377 (2017).

36. Arroyo-Currás, N. \& Bard, A. J. Iridium oxidation as observed by surface interrogation scanning electrochemical microscopy. J. Phys. Chem. C 119, 8147-8154 (2015).

37. Ahn, H. S. \& Bard, A. J. Surface interrogation scanning electrochemical microscopy of $\mathrm{Nil}-\mathrm{xFexOOH}(0<\mathrm{x}<0.27)$ oxygen evolving catalyst: kinetics of the "fast" iron sites. J. Am. Chem. Soc. 138, 313-318 (2016).

38. Huang, E. W. et al. A study of lattice elasticity from low entropy metals to medium and high entropy alloys. Scr. Mater. 101, 32-35 (2015).

39. El-Seidy, A. M. \& Eissa, A. A. The effect manganese concentration on the corrosion resistance and physical properties of $\mathrm{Zn}-\mathrm{Ni}-\mathrm{Mn}$ alloy films produced by electrodeposition. Int. J. Electrochem. Sci. 10, 6273-6287 (2015).

40. Zangari, G. Electrodeposition of alloys and compounds in the era of microelectronics and energy conversion technology. Coatings 5, 195-218 (2015).

41. Sheng, W., Gasteiger, H. A. \& Shao-Horn, Y. Hydrogen oxidation and evolution reaction kinetics on platinum: acid vs alkaline electrolytes. J. Electrochem. Soc. 157, B1529-B1536 (2010).

42. Jiao, F. \& Frei, H. Nanostructured cobalt oxide clusters in mesoporous silica as efficient oxygen-evolving catalysts. Angew. Chem. Int. Ed. 48, 1841-1844 (2009).

43. Gong, L., Chng, X. Y. E., Du, Y., Xi, S. \& Yeo, B. S. Enhanced catalysis of the electrochemical oxygen evolution reaction by iron(III) ions adsorbed on amorphous cobalt oxide. ACS Catal. 8, 807-814 (2018).

44. Godwin, I. J. \& Lyons, M. E. G. Enhanced oxygen evolution at hydrous nickel oxide electrodes via electrochemical ageing in alkaline solution. Electrochem. Commun. 32, 39-42 (2013).

45. Burke, M. S., Enman, L. J., Batchellor, A. S., Zou, S. \& Boettcher, S. W. Oxygen evolution reaction electrocatalysis on transition metal oxides and (oxy) hydroxides: activity trends and design principles. Chem. Mater. 27, 7549-7558 (2015).

46. Abreu-Sepulveda, M. A. et al. The influence of Fe substitution in lanthanum calcium cobalt oxide on the oxygen evolution reaction in alkaline media. $J$. Electrochem. Soc. 163, F1124-F1132 (2016).

47. Sharma, A. S., Yadav, S., Biswas, K. \& Basu, B. High-entropy alloys and metallic nanocomposites: processing challenges, microstructure development and property enhancement. Mat. Sci. Eng. R 131, 1-42 (2018).

48. Goldsmith, Z. K. et al. Characterization of NiFe oxyhydroxide electrocatalysts by integrated electronic structure calculations and spectroelectrochemistry. Proc. Natl Acad. Sci. USA 114, 3050-3055 (2017).

49. McCrory, C. C. L., Jung, S. H., Peters, J. C. \& Jaramillo, T. F. Benchmarking heterogeneous electrocatalysts for the oxygen evolution reaction. J. Am. Chem. Soc. 135, 16977-16987 (2013).

50. Chen, G.-F. et al. Efficient and stable bifunctional electrocatalysts $\mathrm{Ni} / \mathrm{NixMy}$ $(\mathrm{M}=\mathrm{P}, \mathrm{S})$ for overall water splitting. Adv. Funct. Mater. 26, 3314-3323 (2016).

51. Jin, W., Du, H., Zheng, S., Xu, H. \& Zhang, Y. Comparison of the oxygen reduction reaction between $\mathrm{NaOH}$ and $\mathrm{KOH}$ solutions on a Pt electrode: the electrolyte-dependent effect. J. Phys. Chem. B 114, 6542-6548 (2010).

52. Zeng, K. \& Zhang, D. Recent progress in alkaline water electrolysis for hydrogen production and applications. Prog. Energy Combust. Sci. 36, 307-326 (2010).

53. McCrory, C. C. L. et al. Benchmarking hydrogen evolving reaction and oxygen evolving reaction electrocatalysts for solar water splitting devices. J. Am. Chem. Soc. 137, 4347-4357 (2015).

54. Xiao, H., Shin, H. \& Goddard, W. A. Synergy between Fe and Ni in the optimal performance of $(\mathrm{Ni}, \mathrm{Fe}) \mathrm{OOH}$ catalysts for the oxygen evolution reaction. Proc. Natl Acad. Sci. USA 115, 5872-5877 (2018).
55. Barforoush, J. M., Seuferling, T. E., Jantz, D. T., Song, K. R. \& Leonard, K. C. Insights into the active electrocatalytic areas of layered double hydroxide and amorphous nickel-iron oxide oxygen evolution electrocatalysts. ACS Appl Energy Mater. 1, 1415-1423 (2018)

56. Pendergast, A. D., Glasscott, M. W., Renault, C. \& Dick, J. E. One-step electrodeposition of ligand-free PdPt alloy nanoparticles from water droplets: Controlling size, coverage, and elemental stoichiometry. Electrochem. Commun. 98, 1-5 (2019).

57. Jeun, Y. E., Baek, B., Lee, M. W. \& Ahn, H. S. Surfactant-free electrochemical synthesis of metallic nanoparticles via stochastic collisions of aqueous nanodroplet reactors. Chem. Commun. 54, 10052-10055 (2018).

58. Serrà, A. \& Vallés, E. Microemulsion-based one-step electrochemical fabrication of mesoporous catalysts. Catalysts 8, 395 (2018).

\section{Acknowledgements}

J.E.D. gratefully acknowledges UNC Chapel Hill for start-up funds, which supported this work. C.R. acknowledges CNRS for financial support. This work was performed in part at the Chapel Hill Analytical and Nanofabrication Laboratory, CHANL, a member of the North Carolina Research Triangle Nanotechnology Network, RTNN, which is supported by the National Science Foundation, Grant ECCS-1542015, as part of the National Nanotechnology Coordinated Infrastructure, NNCI. This work was performed in part at the Analytical Instrumentation Facility (AIF) at North Carolina State University, which is supported by the State of North Carolina and the National Science Foundation (award number ECCS-1542015). This work made use of instrumentation at AIF acquired with support from the National Science Foundation (DMR1726294). The AIF is a member of the North Carolina Research Triangle Nanotechnology Network (RTNN), a site in the National Nanotechnology Coordinated Infrastructure (NNCI).

\section{Author contributions}

M.W.G. and J.E.D. conceived the research plan and designed experiments with valuable input from C.R. and A.D.P.; M.W.G. performed the material synthesis and characterization via SEM/EDX, HAADF-STEM, and TEM/SAED, and completed the electrocatalytic evaluation; A.D.P. assisted with material synthesis and the collection and processing of SEM/EDX, XPS, and XRD data; S.G. carried out the electrochemical collision experiments on CF-UMEs; A.R.B. assisted with ECSA evaluation and prepared the samples for ICP-MS analysis; A.T.H. provided the spectrophotometric and voltammetric characterization of individual metal salt solutions; M.W.G. and J.E.D. wrote and revised the manuscript with input from C.R., A.D.P., S.G., A.R.B., and A.T.H.; All authors collectively discussed the results and contributed feedback for the manuscript and approved the final version; J.E.D. supervised all aspects of the project.

\section{Additional information}

Supplementary Information accompanies this paper at https://doi.org/10.1038/s41467 019-10303-z.

Competing interests: The authors declare no competing interests.

Reprints and permission information is available online at http://npg.nature.com/ reprintsandpermissions/

Peer review information: Nature Communications thanks Xiaoming Sun and the other, anonymous, reviewer(s) for their contribution to the peer review of this work.

Publisher's note: Springer Nature remains neutral with regard to jurisdictional claims in published maps and institutional affiliations.

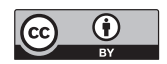

Open Access This article is licensed under a Creative Commons Attribution 4.0 International License, which permits use, sharing, adaptation, distribution and reproduction in any medium or format, as long as you give appropriate credit to the original author(s) and the source, provide a link to the Creative Commons license, and indicate if changes were made. The images or other third party material in this article are included in the article's Creative Commons license, unless indicated otherwise in a credit line to the material. If material is not included in the article's Creative Commons license and your intended use is not permitted by statutory regulation or exceeds the permitted use, you will need to obtain permission directly from the copyright holder. To view a copy of this license, visit http://creativecommons.org/ licenses/by/4.0/.

(C) The Author(s) 2019 